\title{
A Clough-Tocher Type Element Useful for Fourth Order Problems Over Nonpolygonal Domains*
}

\author{
By Lois Mansfield**
}

\begin{abstract}
A new macro-element useful for finite element approximations to fourth order elliptic problems with curved boundaries is presented. An error analysis is given which shows that these elements achieve $O\left(h^{2}\right)$ accuracy in energy.
\end{abstract}

1. Introduction. The purpose of this note is to present a new macro-element useful for finite element approximations to fourth order elliptic problems with curved boundaries. Let $\Omega$ be a closed bounded region of the plane with curved boundary $\Gamma$. Let $\tau$ be a triangulation of $\Omega$ with triangles with at most one curved side along the boundary.

For second order problems a very successful technique for handling curved boundaries is the use of isoparametric transformations. The exact boundary $\Gamma$ of $\Omega$ is replaced by a piecewise polynomial curve $\Gamma_{h}$ and the finite elements are required to satisfy the essential boundary condition $u=0$ on the approximate boundary. On each curved triangle $T$ the finite elements are transforms back to $T$ of polynomial elements on the standard triangle $T_{s}$. The same class of polynomials are used as finite elements on $T_{s}$ as are used in defining the mapping from $T_{s}$ to $T$. Isoparametric elements, however, cannot be used in a conforming finite element method for fourth order problems because one cannot construct a $C^{1}$-coordinate transformation which maps curved triangles onto straight ones. In [7], Zlámal proposes and analyzes a similar procedure but separates the coordinate transformation from the definition of the finite elements.

In our procedure we also separate the coordinate transformation from the definition of the finite elements. We shall assume that twelve parameter CloughTocher cubic macro-elements introduced in [5] are used on interior triangles. We shall use a quadratic polynomial coordinate transformation which means that $\Gamma_{h}$ will be a piecewise quadratic polynomial approximation to the exact boundary $\Gamma$. Although the coordinate transformation is only a $C^{0}$-transformation so that normal derivatives are not preserved, normal derivatives on the edges of the standard triangle can be expressed as easily computed polynomial combinations of tangential and normal deriva-

Received January 24, 1977 ; revised May 3, 1977.

AMS (MOS) subject classifications (1970). Primary 65N30, 65N 15.

* This research was partially supported by the National Science Foundation under grant no. MCS76-06293.

**Current address: Department of Computer Science, University of Kansas, Lawrence, Kansas 66045. 
tives on the edges of the curved triangle. For a quadratic polynomial coordinate transformation and Clough and Tocher elements on interior triangles (which imply that tangential and normal derivatives on the straight edges of curved triangles are quadratic polynomials), these combinations will be cubic polynomials.

Thus, in order to obtain $C^{1}$-finite elements on curved triangles in the region $\Omega_{h}$ with boundary $\Gamma_{h}$ we must use a $C^{1}$-element in $T_{s}$ which has cubic polynomial values and normal derivatives in the edges of $T_{s}$, and choose the defining parameters so that the normal derivatives along edges match the above-mentioned cubic polynomial combinations of tangential and normal derivatives on the straight-sided edges of the curved triangles. An appropriate rational element was constructed in [2] and applied to curved boundaries in fourth order problems in [6].

In this note we construct a new piecewise polynomial macro-element which also has cubic polynomial values and normal derivatives on the edges of $T_{s}$. This element is defined in the next section. In Section 3 we provide a mathematical analysis which shows that our procedure achieves $O\left(h^{2}\right)$ accuracy in energy, i.e. has the same accuracy as Clough-Tocher elements on polygonal domains.

2. Construction of New Macro-Element. On any triangle $T$, the set $T C(T)$ of tricubic polynomials (see [1]) is the set of all polynomials which are cubic along all parallels to the three edges. If $T$ has edges $\lambda_{i}=0, i=0,1,2$, the set of tricubic polynomials on $T$ consists of all cubic polynomials plus linear combinations of two of the quartic polynomials $\lambda_{i}^{3} \lambda_{i+1}+\lambda_{i}^{2} \lambda_{i+1}^{2}, i=0,1,2$. (Here and below all subscripts will be counted mod (3).) Let $T$ be the macro triangle of Figure 1 with vertices $P_{i}, i=0,1,2$, and center of gravity $G$.

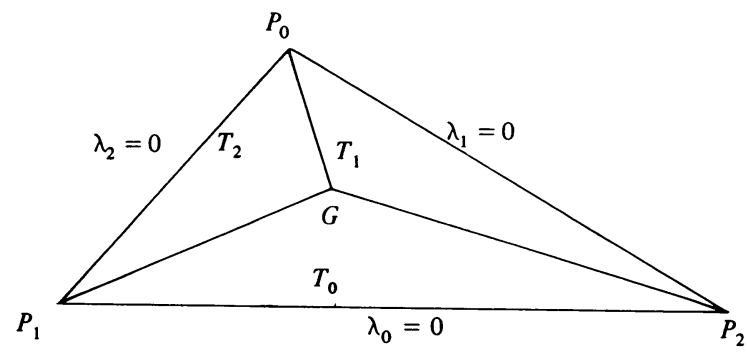

FIGURE 1

Let $\mu_{i, j}=0$ be the equation of the edge opposite $P_{j}$ in the triangle $T_{i}$, normalized so that $\mu_{i, j}\left(P_{j}\right)=1$.

Let $P(T)=\left\{p \in C^{1}(T):\left.p\right|_{T_{i}} \in T C\left(T_{i}\right), i=0,1,2\right\}$.

THEOREM 1. In any triangle $T$ there exists a unique $u \in P(T)$ which has given

(i) values and first partials at each vertex $P_{i}, i=0,1,2$,

(ii) $u_{n}$ and $u_{\tau n}$ at the midpoint of each edge $\lambda_{i}=0$, where $\partial / \partial \tau$ denotes the tangential derivative.

Proof. Let $u$ be a $C^{1}$-piecewise tricubic polynomial with all the conditions (i)(ii) zero. We show that $u \equiv 0$. On $T_{i}, u=p_{i}=\lambda_{i}^{2} q_{i}$, where $q_{i}$ is a quadratic poly- 
nomial which is linear on $T_{i} \cap T_{i+1}$ and $T_{i} \cap T_{i+2}$. Thus

$$
q_{i}=\alpha_{i} \mu_{i, i+2} \mu_{i, i+1}+l_{i}
$$

where $\alpha_{i}$ is some constant and $l_{i}$ is a linear polynomial. If we normalize $\lambda_{i}=0$ so that $\lambda_{i}(G)=1, i=0,1,2$, then $\lambda_{i}=\lambda_{i+1}$ on $T_{i} \cap T_{i+1}$, and $p_{i}=p_{i+1}$ on $T_{i} \cap T_{i+1}$ implies that $q_{i}=q_{i+1}$ on $T_{i} \cap T_{i+1}$ which in turn implies that $l_{i}=l_{i+1}$ on $T_{i} \cap T_{i+1}$. On $T_{i} \cap T_{i+1}$

$$
2 \lambda_{i} \nabla \lambda_{i} q_{i}-2 \lambda_{i+1} \nabla \lambda_{i+1} q_{i+1}+\lambda_{i}^{2} \nabla q_{i}-\lambda_{i+1}^{2}=0
$$

or equivalently

$$
2 \nabla\left(\lambda_{i}-\lambda_{i+1}\right) q_{i}+\lambda_{i}\left(\nabla q_{i}-\nabla q_{i+1}\right)=0 \quad \text { on } T_{i} \cap T_{i+1} .
$$

Thus $\lambda_{i}\left(P_{i+2}\right)=0$ implies that $q_{i}\left(P_{i+2}\right)=l_{i}\left(P_{i+2}\right)=0$, which together with $l_{i}=$ $l_{i+1}$ on $T_{i} \cap T_{i+1}$ and a similar result on $T_{i} \cap T_{i+2}$ implies that $l_{i}=\beta \lambda_{i}$, where $\beta$ is some constant. Thus, we have

$$
\nabla p_{i}=\alpha_{i} \lambda_{i}^{2} \mu_{i, i+2} \nabla \mu_{i, i+1}+3 \beta \lambda_{i}^{2} \nabla \lambda_{i} \quad \text { on } T_{i} \cap T_{i+1} .
$$

The continuity of the $\nabla p_{i}$ at $G$ implies that $\beta=0$. At the midpoint of $T_{i} \cap T_{i+1}$, $\lambda_{i}=\lambda_{i+1}=\mu_{i, i+2}=\mu_{i+1, i+2}=1 / 2$. Thus, from (1) we obtain the relations $\alpha_{i} \nabla \mu_{i, i+1}=\alpha_{i+1} \nabla \mu_{i+1, i}$. Since $\nabla \mu_{i, i+1}=-\nabla \mu_{i+1, i}$, it follows that

$$
\alpha_{0}=-\alpha_{1}, \quad \alpha_{1}=-\alpha_{2}, \quad \alpha_{2}=-\alpha_{0},
$$

which is possible only if $\alpha_{0}=\alpha_{1}=\alpha_{2}=0$. This completes the proof.

We give a cardinal basis for $P\left(T_{s}\right)$ with respect to the interpolation conditions of Theorem 1. Let $P_{0}=(0,0), P_{1}=(1,0), P_{2}=(0,1)$, and let $M_{i}$ be the midpoint of $\lambda_{i}=0$. An element $g \in P\left(T_{s}\right)$ can be expressed as

$$
\begin{aligned}
g= & \sum_{i=0}^{2}\left(g\left(P_{i}\right) \phi_{i+1}+g_{x}\left(P_{i}\right) \phi_{i+4}+g_{y}\left(P_{i}\right) \phi_{i+7}\right) \\
& +g_{y}\left(M_{2}\right) \phi_{10}+g_{x y}\left(M_{2}\right) \phi_{11}+g_{x}\left(M_{1}\right) \phi_{12}+g_{x y}\left(M_{1}\right) \phi_{13} \\
& +\left(g_{x}\left(M_{0}\right)+g_{y}\left(M_{0}\right)\right) \phi_{14}+\left(g_{x x}\left(M_{0}\right)-g_{y y}\left(M_{0}\right)\right) \phi_{15}
\end{aligned}
$$

where

$$
\begin{aligned}
& \phi_{1}=3(1-x-y)^{2}-2(1-x-y)^{3}+3 \epsilon_{1} / 2+3 \epsilon_{2} / 2 \\
& \phi_{2}=3 x^{2}-2 x^{3}+3 / 4 \epsilon_{0} \\
& \phi_{3}=3 y^{2}-2 y^{3}+3 / 4 \epsilon_{0} \\
& \phi_{4}=x(1-x-y)^{2}-1 / 4 \epsilon_{1}+1 / 2 \epsilon_{2}+\delta_{1} \\
& \phi_{5}=x^{3}-x^{2}+1 / 4 \epsilon_{0}-1 / 2 \delta_{1} \\
& \phi_{6}=x y^{2}-1 / 4 \epsilon_{1}-3 / 4 \epsilon_{2}-\delta_{1}+1 / 2 \delta_{0} \\
& \phi_{7}=y(1-x-y)^{2}-1 / 4 \epsilon_{2}+1 / 2 \epsilon_{1}+\delta_{2}
\end{aligned}
$$




$$
\begin{aligned}
& \phi_{8}=x^{2} y-1 / 4 \epsilon_{2}-3 / 4 \epsilon_{1}-\delta_{2}-1 / 2 \delta_{0}, \\
& \phi_{9}=y^{3}-y^{2}+1 / 4 \epsilon_{0}+1 / 2 \delta_{0}, \\
& \phi_{10}=\epsilon_{2} \\
& \phi_{11}=\delta_{2} \\
& \phi_{12}=\epsilon_{1} \\
& \phi_{13}=\delta_{1}, \\
& \phi_{14}=-1 / 2 \epsilon_{0}, \\
& \phi_{15}=1 / 2 \delta_{0},
\end{aligned}
$$

where

$$
\epsilon_{i}= \begin{cases}\frac{2}{9} \lambda_{i}^{2}-\frac{14}{81} \lambda_{i}^{3}+\frac{4}{3} \lambda_{i} \mu_{i, i+2} \mu_{i, i+1} \quad \text { on } T_{i} \\ \frac{4}{81} \lambda_{i+2}^{2}+\frac{14}{81} \lambda_{i+2}^{2} \mu_{i+2, i+1}-\frac{4}{81} \lambda_{i+2}^{2} \mu_{i+2, i} & \text { on } T_{i+2} \\ \frac{4}{81} \lambda_{i+1}^{2}-\frac{4}{81} \lambda_{i+1}^{2} \mu_{i+1, i}+\frac{14}{81} \lambda_{i+1}^{2} \mu_{i+1, i+2} & \text { on } T_{i+1}\end{cases}
$$

and

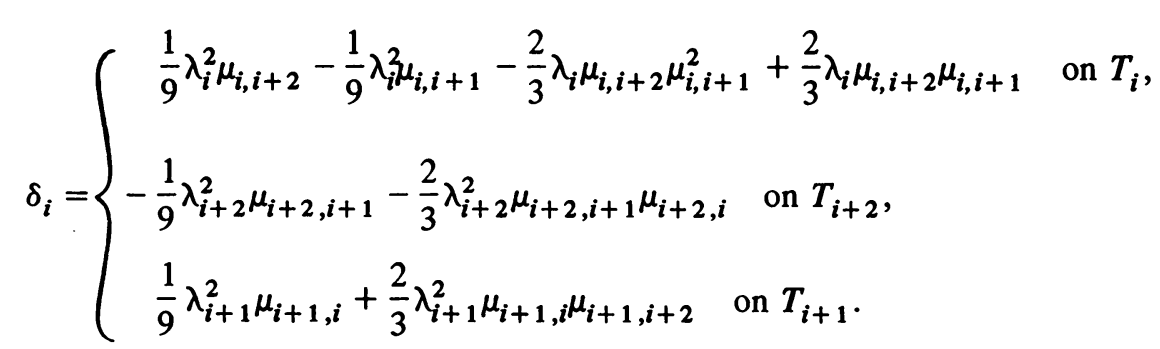

3. Error Analysis. In this section we show how the macro-element constructed in the previous section can be used in the finite element method applied to fourth order elliptic equations defined on nonpolygonal regions $\Omega$ of the plane, and we analyze the error. Throughout we take the boundary conditions to be the homogeneous Dirichlet conditions.

$$
u=u_{n}=0 \text { on } \Gamma \text {. }
$$

Let $T$ be the curved triangle shown in Figure 2 with the curved side given by the quadratic polynomial curve approximation to the curve $\Gamma$ which interpolates $\Gamma$ at $P_{1}, P_{2}$ and a point $M_{0}$ between $P_{1}$ and $P_{2}$.

The standard triangle $T_{s}$ with vertices $Q_{0}=(0,0), Q_{1}=(1,0)$ and $Q_{2}=(0,1)$ is mapped onto $T$ by the mapping

$$
\mathrm{x}=(1-s-t) P_{0}+(s-2 s t) P_{1}+(t-2 s t) P_{2}+4 s t M_{0},
$$

where $\mathrm{x}=(x, y)$. Let $E_{i}$ be the edge opposite the vertex $P_{i}$ in $T$ and let $E_{i}$ be the edge opposite the vertex $Q_{i}$ in $T_{s}$. We want to construct finite elements $\hat{\Psi}(s, t)$ whose 


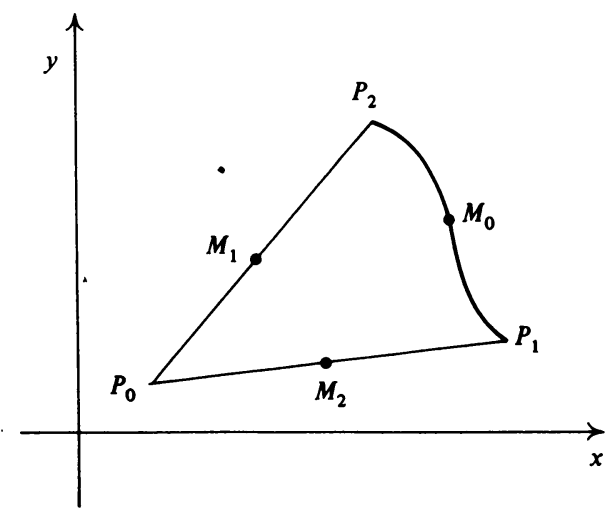

FigURE 2

transforms back to $T$ defined by

$$
\Psi(x, y)=\Psi(x(s, t), y(s, t))=\hat{\Psi}(s, t)
$$

match on the straight edges $E_{1}$ and $E_{2}$, given cubic polynomial values $f_{i}$ and given quadratic polynomial normal derivatives $g_{i}$ which come from the use of Clough-Tocher elements in interior triangles. Throughout we define $f_{2}$ and $g_{2}$ by

$$
\begin{gathered}
f_{2}(s)=u(x(s, 0), y(s, 0)), \\
g_{2}(s)=\frac{\partial u}{\partial x}(x(s, 0), y(s, 0)) \frac{\partial y}{\partial s}-\frac{\partial u}{\partial y}(x(s, 0), y(s, 0)) \frac{\partial x}{\partial s},
\end{gathered}
$$

with similar definitions for $f_{i}$ and $g_{i}, i=0,1$. Thus $f_{2}^{\prime}$ and $g_{2}$ are the tangential and normal derivative along $E_{2}$ scaled by the length of $E_{2}$. The normal derivative $\hat{g}_{i}$ on the edge $E_{i}$ of $T_{s}$ under the mapping (3) is given by

$$
\hat{g}_{i}=\rho_{i} f_{i}^{\prime}+\sigma_{i} g_{i} \text {, }
$$

where for $i=1,2$, the functions $\rho_{i}$ and $\sigma_{i}$ are linear polynomials. The functions $\rho_{0}$ and $\sigma_{0}$ are rational functions but they do not actually enter into our computations since we shall take $f_{0}$ and $g_{0}$ to be zero in order to match the homogeneous Dirichlet boundary conditions. We choose our finite elements $\hat{\Psi}(s, t)$ to be elements of $P\left(T_{s}\right)$ which have zero values and normal derivatives on $E_{0}$ and which match given cubic polynomial values $\hat{f}_{i}=f_{i}$ and the normal derivatives $\hat{g}_{i}$ given by (4) on the edges $E_{1}$ and $E_{2}$.

In the triangulation $\tau$, let $h$ be the length of the longest side and let $\alpha$ be the smallest angle. We assume that

$$
\alpha \geqslant \alpha_{0}>0,
$$

where $\alpha_{0}$ is an absolute constant not depending on $h$. It can be shown that under the assumption (5)

$$
c_{1} \leqslant\left|\rho_{i}^{(j)}\right|, \quad\left|\sigma_{i}^{(j)}\right| \leqslant c_{2}, \quad i=0,1,2, j=0,1,
$$

where $c_{1}$ and $c_{2}$ are absolute constants depending only on $\sin \alpha_{0}$.

In order to show that our procedure for curved domains has the same order of accuracy in the energy norm as the use of Clough-Tocher elements on polygonal do- 
mains, it is sufficient (see $\left[6\right.$, Section 2]) to show that for some $v_{h}$ in our finite element space $S_{h} \subset H_{0}^{2}\left(\Omega_{h}\right)$

$$
\left\|\tilde{u}-v_{h}\right\|_{H^{2}\left(\Omega_{h}\right)} \leqslant K h^{2}
$$

where $K$ is independent of $h$, and $\tilde{u}$ is the extension of the exact solution $u$ to $\Omega_{h}$ (in the case that $\Omega_{h} \not \subset \Omega$ ). On interior triangles we take $v_{h}$ to be the Clough-Tocher interpolant analyzed in [3]. On curved triangles we take $v_{h}$ to be the interpolant $\tilde{u}_{I}$ to $\tilde{u}$ defined as follows. Suppose $\tilde{u}$ has values $f_{j}$ and normal derivatives $g_{j}$ on the edges of $T$. Let $H C f_{j}$ be the cubic polynomial interpolant to $f_{j}$ with respect to values and tangential derivatives at the vertices, and let $Q U g_{j}$ be the quadratic polynomial interpolant to values of $g_{j}$ at the vertices and at $M_{j}$. We define $\tilde{u}_{I}$ to be the transform under (3) of the element $\hat{u}_{I}$ of $P\left(T_{s}\right)$ which has cubic polynomial values $\bar{f}_{j}=H C f_{j}$ and cubic polynomial normal derivatives $\bar{g}$ given by

$$
\bar{g}_{j}=\rho_{i}\left(H C f_{j}\right)^{\prime}+\sigma_{j} Q U g_{j}, \quad j=0,1,2 .
$$

Note that $H C f_{0}=Q U g_{0}=0$ so that $v_{h} \subset H_{0}^{2}(\Omega)$.

TheOREM 2. Let $\tilde{u} \in C^{4}(T)$ and let $\tilde{u}_{I}$ be the interpolant to $\tilde{u}$ defined in the preceding paragraph. Then there exists a constant $C$ independent of $u$ and $h$ such that

$$
\left|\tilde{u}-\tilde{u}_{I}\right|_{k, \infty, T} \leqslant C h^{4-k}\|\tilde{u}\|_{w^{4, \infty}(T)}, \quad 0 \leqslant|k| \leqslant 2 .
$$

Proof. Let $e=\tilde{u}-\tilde{u}_{I}$ and $\hat{e}(s, t)=e(x(s, t), y(s, t))$. By Theorem 2 of [6], there exist constants independent of $h$ such that

$$
C_{1} h^{2} \leqslant|J(s, t)| \leqslant C_{2} h^{2},
$$

where $J$ is the Jacobian of the mapping (3). In addition,

$$
\begin{aligned}
& D^{i} x(s, t)=O\left(h^{|i|}\right), \quad D^{i} y(s, t)=O\left(h^{|i|}\right) \quad \text { all } i, \\
& D^{i} s(x, y)=O\left(h^{-|i|}\right), \quad D^{i} t(x, y)=O\left(h^{-|i|}\right), \quad 1 \leqslant|i| \leqslant 2 .
\end{aligned}
$$

Thus

$$
\left|D^{\alpha} e(x, y)\right| \leqslant K h^{-k} \sum_{|\beta| \leqslant k}\left|D^{\beta} \hat{e}(s, t)\right|, \quad|\alpha|=k .
$$

We write $\hat{e}(s, t)$ as $\hat{e}=\hat{u}-\pi \hat{u}+\pi \hat{u}-\hat{u}_{I}$, where $\pi \hat{u}$ is the unique element of $P\left(T_{s}\right)$ which interpolates $\hat{u}$ with respect to the conditions of Theorem 1 . The method used by Ciarlet in the error analysis in [3] can be applied directly (except for a change in norm) to show that

$$
\left|D^{\beta} \hat{u}(s, t)-D^{\beta}(\pi \hat{u})(s, t)\right| \leqslant K|\hat{u}|_{4, \infty, T_{s}} \leqslant K^{\prime} h^{4}\|\tilde{u}\|_{W^{4, \infty}(T)} .
$$

To complete the proof, we must show that

$$
\left|D^{\beta}(\pi \hat{u})(s, t)-D^{\beta} \hat{u}_{I}(s, t)\right| \leqslant K h^{4}\|\tilde{u}\|_{W^{4, \infty}(T)},
$$

for some positive constant $K$. 
Note that $D^{\alpha} \hat{u}_{I}$ and $D^{\alpha} \pi \hat{u}, 0 \leqslant|\alpha| \leqslant 1$, agree at the vertices of $T_{s}$ and on the edge $E_{1}$. Thus

$$
D^{\beta} \pi u(s, t)-D^{\beta} \hat{u}_{I}(s, t)=\sum_{i=1}^{4} a_{i} D^{\beta} F_{i}(s, t),
$$

where $F_{1}, F_{2}$ are the cardinal functions associated with the linear functionals $\partial u\left(R_{i}\right) / \partial n$, where the $R_{i}$ are the midpoints of the edges $E_{1}$ and $E_{2}$ of $T_{s}$, and $F_{i+2}$, $i=1,2$, are the cardinal functions associated with the linear functionals $\partial^{2} u\left(R_{i}\right) / \partial n \partial \tau$. Thus

$$
\begin{aligned}
a_{i}= & \rho_{i}(1 / 2)\left[f_{i}^{\prime}(1 / 2)-\left(H C f_{i}\right)^{\prime}(1 / 2)\right], \\
a_{i+2}= & \rho_{i}^{\prime}(1 / 2)\left[f_{i}^{\prime}(1 / 2)-\left(H C f_{i}\right)^{\prime}(1 / 2)\right]+\rho_{i}(1 / 2)\left[f_{i}^{\prime \prime}(1 / 2)-\left(H C f_{i}\right)^{\prime \prime}(1 / 2)\right] \\
& +\sigma_{i}(1 / 2)\left[g_{i}^{\prime}(1 / 2)-\left(Q U g_{i}\right)^{\prime}(1 / 2)\right], \quad i=1,2 .
\end{aligned}
$$

The functions $D^{\beta} F_{i}(s, t), 0 \leqslant|\beta| \leqslant 2$, can be uniformly bounded. By the Peano Kernel Theorem,

and

$$
\begin{aligned}
\left|f_{i}^{(j)}(1 / 2)-H C f_{i}^{(j)}(1 / 2)\right| & \leqslant K \max _{s \in[0,1]}\left|f_{i}^{(4)}(s)\right| \\
& \leqslant K|\hat{u}|_{4, \infty, T_{s}} \leqslant K^{\prime} h^{4}|\tilde{u}|_{4, \infty, T, \quad j=1,2,}
\end{aligned}
$$

$$
\begin{aligned}
\left|g_{i}^{\prime}(1 / 2)-\left(Q U g_{i}\right)^{\prime}(1 / 2)\right| & \leqslant K \max _{s \in[0,1]}\left|g_{i}^{(3)}(s)\right| \\
& \leqslant K|\hat{u}|_{4, \infty, T_{s}} \leqslant K^{\prime} h^{4}|\tilde{u}|_{4, \infty, T} .
\end{aligned}
$$

This combined with (6) establishes (11) and proves the theorem.

The bound (7) follows from Theorem 2.

Similarly to isoparametric elements for second order problems, numerical integration is required to evaluate the integrals involving partial derivatives. The analysis of [4, Chapter 11] and [7] cannot directly be extended to apply to our finite elements since we have not shown the convergence of any $v \in S_{h}$ to $\tilde{u}$ in $H^{m}\left(T_{i}\right)$ on each subtriangle $T_{i}$ for $m>2$. The analysis of [6], however, can be applied to show that $O\left(h^{2}\right)$ accuracy in energy is preserved if an integration rule exact for quintic polynomials is used for each subtriangle of each curved triangle. This is in contrast to the fact that for equations with constant coefficients, such as the biharmonic problem, a rule exact for quadratic polynomials is sufficient for subtriangles of interior triangles.

One should expect greater accuracy to be required on curved triangles, however, since finite elements associated with curved triangles are tricubic on each subtriangle of $T_{s}$ rather than cubic and second partials in $x$ and $y$ are transformed to a sum of first and second partials in $s$ and $t$ by the mapping (3), rather than a sum of second partials alone. This is in contrast to second order problems.

ICASE

NASA Langley Research Center

Hampton, Virginia 23665 
1. G. BIRKHOFF, "Tricubic interpolation in triangles," Proc. Nat. Acad. Aci. U.S.A., v. 68, 1971, pp. $1162-1164$.

2. G. BIRKHOFF \& L. MANSFIELD, “Compatible triangular finite elements," J. Math. Anal. Appl, v. 47, 1974, pp. 531-553.

3. P. G. CIARLET, "Sur l'elément de Clough et Tocher," Rev. Française Automat. Informat. Recherche Opérationnelle Sér. Rouge, v. 8, 1974, pp. 19-27.

4. P. G. CIARLET, Numerical Analysis of the Finite Element Method, University of Montreal Press, 1976.

5. R. W. CLOUGH \& J. L. TOCHER, "Finite element stiffness matrices for analysis of plates in bending," Conferences on Matrix Methods in Structural Mechanics, Wright-Patterson A. F. B., Ohio, 1965.

6. L. MANSFIELD, "Approximation of the boundary in the finite element solution of fourth order problems," SIAM J. Numer. Anal. (To appear.)

7. M. ZLÁMAL, "Curved elements in the finite element method. I, II," SIAM J. Numer. Anal, v. 10, 1973, pp. 229-240; v. 11, 1974, pp. 347-362. 